University of Wollongong

Research Online

Faculty of Engineering and Information

Faculty of Engineering and Information

Sciences - Papers: Part A

Sciences

2016

\title{
Theoretical and experimental study on a compliant flipper-leg during terrestrial locomotion
}

\author{
Tao Fang \\ University of Science and Technology of China \\ Youcheng Zhou \\ University of Science and Technology of China \\ Shikun Li \\ University of Science and Technology of China \\ Min Xu \\ University of Science and Technology of China \\ Haiyi Liang \\ University of Science and Technology of China
}

See next page for additional authors

Follow this and additional works at: https://ro.uow.edu.au/eispapers

Part of the Engineering Commons, and the Science and Technology Studies Commons

Research Online is the open access institutional repository for the University of Wollongong. For further information contact the UOW Library: research-pubs@uow.edu.au 


\title{
Theoretical and experimental study on a compliant flipper-leg during terrestrial locomotion
}

\author{
Abstract \\ An amphibious robot with straight compliant flipper-legs can conquer various amphibious environments. \\ The robot can rotate its flipper-legs and utilize their large deflection to walk on rough terrain, and it can \\ oscillate the straight flipper-legs to propel itself underwater. This paper focuses on the dynamics of the \\ compliant straight flipper-legs during terrestrial locomotion by modeling its deformation dynamically with \\ large deflection theory and simulating it to investigate the parameters of locomotion such as trajectory, \\ velocity, and propulsion. To validate the theoretical model of dynamic locomotion, a single-leg \\ experimental platform is used to explore the flipper-legs in motion with various structural and kinematic \\ parameters. Furthermore, a robotic platform mounting with four compliant flipper-legs is also developed \\ and used to experiment with locomotion. The trajectories of the rotating axle of the compliant flipper-leg \\ during locomotion were approximately coincidental in simulation and in experiments. The speed of \\ locomotion and cost of transport during locomotion were explored and analyzed. The performance of \\ different types of compliant flipper-legs during locomotion shows that varying the degrees of stiffness \\ will have a significant effect on their locomotion. The dynamic model and analysis of the compliant \\ flipper-leg for terrestrial locomotion facilitates the ability of amphibious robots to conquer complex \\ environments.

\section{Disciplines} \\ Engineering | Science and Technology Studies

\section{Publication Details} \\ Fang, T., Zhou, Y., Li, S., Xu, M., Liang, H., Li, W. \& Zhang, S. (2016). Theoretical and experimental study on a \\ compliant flipper-leg during terrestrial locomotion. Bioinspiration and Biomimetics, 11 (5), \\ 056005-1-056005-12.
}

\section{Authors}

Tao Fang, Youcheng Zhou, Shikun Li, Min Xu, Haiyi Liang, Weihua Li, and Shiwu Zhang 


\title{
Theoretical and Experimental Study on a Compliant Flipper-leg during Terrestrial
}

\section{Locomotion}

\author{
Tao Fang ${ }^{1}$, Youcheng Zhou ${ }^{1}$, Shikun $\mathrm{Li}^{2}$, Zhuo Chen ${ }^{1}$, Min Xu ${ }^{1}$, Weihua $\mathrm{Li}^{3}$, Shiwu Zhang ${ }^{1}$ \\ ${ }^{1}$ Dept. of Precision Machinery and Precision Instrumentation, University of Science and Technology of China, Hefei, Anhui, 230026, China \\ ${ }^{2}$ Dept. of Modern Mechanics, University of Science and Technology of China, Hefei, Anhui, 230026, China \\ ${ }^{3}$ School of Mechanical, Materials and Mechatronic Engineering, University of Wollongong, Wollongong, NSW 2522, Australia
}

\begin{abstract}
An amphibious robot with straight compliant flipper-legs can conquer various amphibious environments. The robot can rotate its flipper legs and utilise their large deflection to walk on rough terrain, and it can oscillate the straight flipper legs to propel itself underwater. This paper focuses on the dynamics of the compliant straight flipper legs during terrestrial locomotion by modelling its deformation dynamically with large deflection theory and simulating it to investigate the parameters of locomotion such as trajectory, velocity, and propulsion. To validate the theoretical model of dynamic locomotion, a single-leg experimental platform is used to explore the flipper legs in motion with various structural and kinematic parameters. Furthermore, a robotic platform mounting with four compliant flipper-legs is also developed and used to experiment with locomotion. The trajectories of the rotating axle of the compliant flipper leg during locomotion were approximately coincidental in simulation and in experiments. The speed of locomotion and cost of transport (COT) during locomotion were explored and analysed. The performance of different types of compliant flipper-legs during locomotion shows that varying the degrees of stiffness will have a significant effect on their locomotion. The dynamic model and analysis of the compliant flipper-leg for terrestrial locomotion facilitates the ability of amphibious robots to conquer complex environments.
\end{abstract}

Keywords: compliant flipper-leg, amphibious robot, mechanical analysis, single-leg platform, four-leg-robot platform

driving modules. Hence, a simpler mechanism of transformable propulsion for the amphibious robot is needed

\section{Introduction}

Amphibious robots perform important roles in many civilian and military applications such as navigation shores, clearing mines, and mapping terrain [1]. However, different types of amphibious robots have been developed for different roles, such as salamander-like robot [2],[3], ACM series robots [4], and turtle-like robots [5]. To improve their performance in terrestrial and underwater tasks, amphibious robots need to be stable and able to adapt to various terrains and water environments. Nowadays, researchers are proposing approaches that will enable robots to smoothly and efficiently switch between different operational modes on terrestrial and aquatic environments. We have developed an actively transformable flipper-leg capable of swimming under water and walking on terrain, and an amphibious robot AmphiHex-I where the mechanism is a propulsion unit that has been assembled and implemented [6]-[8]. AmphiHex-I has six flipper-legs with embedded steel plates and cables that can transform the flipper-legs between straight flippers and curved legs by loosening or tightening the cables. However, this active transformation needs extra motors to move the cables, which increases the complexity of the control strategy and for field applications.

Many other robots, as well as AmphiHex-I use soft structures for the propulsion unit and the connector or body, such as the arms of an octopus-like robot or compliant joints in artificial fingers, and Softworms [9][11] Inspired by these soft structures, we developed a compliant flipper leg that can act as the propulsion unit. This compliant flipper-like leg is designed as a straight plate made from an elastic material that can be transformed passively [12]. This flipper-leg can propel the robot underwater like turtle's flippers, and also bend to a curved shape for terrestrial locomotion when normal and tangential forces exerted by different terrains applied. Thus, this compliant flipper-leg has a similar function as AmphiHex-I, and thus has a simpler structure, a simplified driving module, and an easier control strategy.

This flipper-leg has a two-fold compliance: (1) it can be used as a flipper and oscillate to propel underwater, and (2) it can bend into a curved leg during locomotion on different terrain. Compliant flippers mounted on robots as propulsion units to mimic animal flippers has been applied and verified in many underwater robots [13]-[17]. However, the ability of straight compliant flipper legs to propel an amphibious robot on different terrain still needs further work. 
The dynamics of a compliant flipper-leg must be examined in order to understand their propulsive ability on different terrains. However, modelling the dynamics of a compliant flipper leg means simplifying its deformation and the complex interaction between the flipper leg and terrain. This deformation is difficult to model due to its nonlinearity and multi- dimensionality. Aribert has contributed a great deal to the analysis and application of compliant legs [18] by focusing on compliant legs that mainly consist of rigid and soft parts that have theoretically proved to be stable, controllable, and efficient due to compliance [19]. Since a compliant leg made from soft material, as a pseudo rigid body model (PRBM), has proved a successful way of modelling a compliant beam with large deflections [20]-[22], PRBM regards a compliant beam as a combination of two rigid bars and a torque spring set at a suitable position, while neglecting any nonlinearity of the material in large deformations. As with PRBM, PRBM 3R divides a compliant beam into three rotating springs and four bars that results in a more accurate but more complex model than PRBM [23]. A rolling spring loaded inverted pendulum (RLISP) is suitable for curved-leg, like that of REHX, because it divides the leg into a straight rigid bar, a curved rigid bar, and a rotating spring [24],[25]. However, it is hard to derive an explicit solution because these theoretical models are too complex, so Finite Element Analysis (FEA) is a good choice to cover mechanical problems that include a credible nonlinearity and the complex interaction of parts with each other [26],[27].

In this paper we aim to explore the feasibility and performance of a compliant flipper leg during locomotion on terrain by theoretical modelling and experimental validation. We used large deflection equations once used to verify PRBM, to model the compliant leg, and set up a finite element model to simulate the entire motion of the leg to obtain more parameters. With these methods, we can explore movements such as forward speed, fluctuations in height, propulsion efficiency, and the design of the compliant legs. The theoretical results were validated by experiments with moving platform, and moving robots. This study facilitates implementing amphibious robots to conquer various complex environments.

The reminder of the paper is organised as follows. Section 2 introduces the leg locomotion platform, the theoretical model of the compliant flipper-leg, and the robot locomotion platform. Section 3 presents the performance of propulsion from simulation and experiment, the special design of the compliant legs, and Section 4 presents the conclusion.

\section{Experimental System and Mechanical Model}

In order to explore the feasibility and performance of straight compliant leg moving over terrain, we developed a single leg locomotion platform, a theoretical model of the compliant leg, and a robot locomotion platform to conduct a theoretical and experimental analysis.

\subsection{Single Leg Locomotion Platform}

A single leg locomotion platform was developed to explore the movement of compliant flipper legs. Figure 1a shows a simplified draft of the locomotion platform; it consists of a horizontal cylindrical slide, two vertical cylindrical slides, a vertical displacement sensor, and a locomotion unit. The cylindrical slides provide horizontal and vertical translational freedoms for the locomotion unit; the propulsion unit also includes a driving unit and a compliant flipper leg. The leg is made from polyurethane, which is widely used as a super elastic material. The driving unit contains a MAXON motor to drive the flipper leg, a gear box, and a torque sensor, and the coordinate is set at the centre of the flipper leg's rotation. Fig. $1 b$ is a series of photographs of the leg during terrestrial motion. The red pointer at the front of the drive unit is used to measure the angle of rotation of the flipper leg. The MAXON motor is controlled to vary the speed of rotation in a clockwise direction, and a weight is used to adjust the payload of the driving unit. The load applied vertically to the compliant leg is $2 \mathrm{~kg}$, and $12.36 \mathrm{~kg}$ applied horizontally. The vertical load means the weight of a driving unit capable of moving in a vertical direction, while the horizontal load includes two vertical slide ways, two horizontal slide ways, and the driving unit and sensors that can move in a horizontal direction. The leg is $310 \mathrm{~mm}$ long $\mathrm{x} 55 \mathrm{~mm}$ wide $\mathrm{x} 15 \mathrm{~mm}$ deep; the leg weighs $76.7 \mathrm{~g}$ and Young's module is $25 \mathrm{Mpa}$.

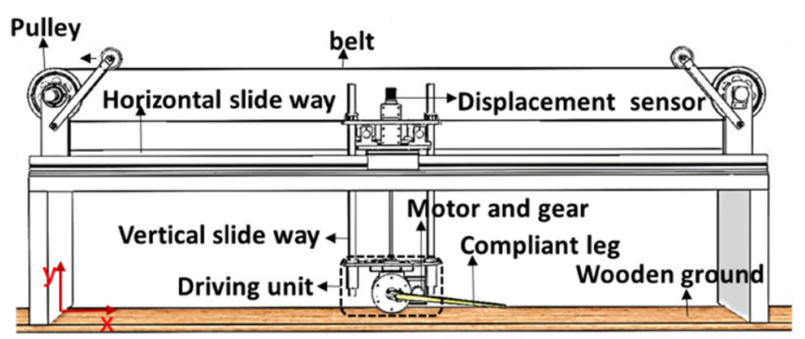

(a) 


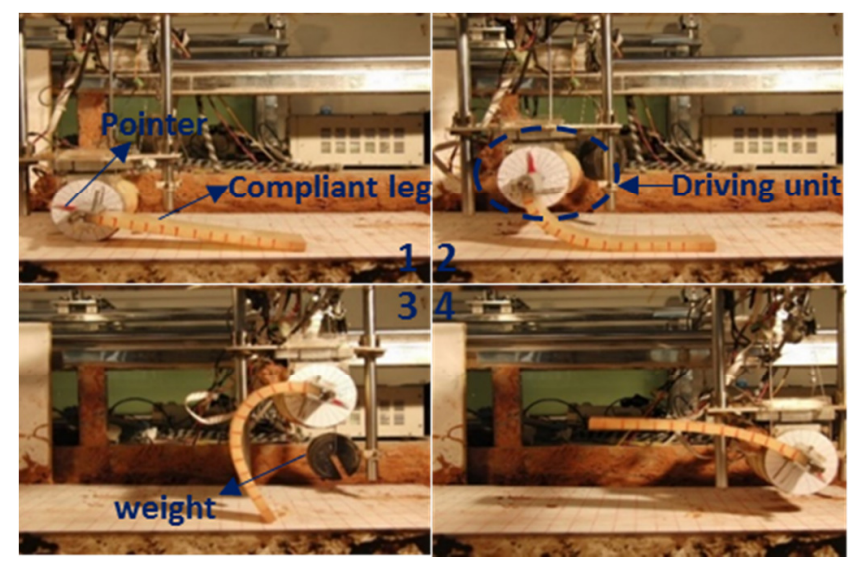

(b)

Figure 1. Single leg locomotion platform. (a) Draft of the locomotion platform. (b) Snapshots of the compliant leg during one cycle in locomotion.

The process by which the compliant leg is propelled on the locomotion platform is shown in Figure 1 (b). Here the leg is straight (see Figure 1.b (1)), but when the driving unit starts the leg bends into a curved shape under the driving load (see Figure 1.b (2)), while the driving unit is pushed forward by the flipper leg. When the flipper leg leaves the ground the driving unit drops down freely (see Figure .1b (3) and 1b (4)). During locomotion, the flipper leg bends continuously, an action that determines how well the propulsive unit can move itself along the platform.

\subsection{Model of Compliant Flipper Leg}

In PRBM or similar models, transforming part of the leg to be analysed is simplified into bars of fixed lengths with torsion springs, but the length of that part of the flipper-leg that contacts the ground varies, which means the length of the bent part also varies; this means that modelling the leg as two bars with fixed lengths and rotation spring in a fixed position is very difficult, so the transforming part of the leg is regarded as a cantilever capable of large deflection, as shown in Figure 2.

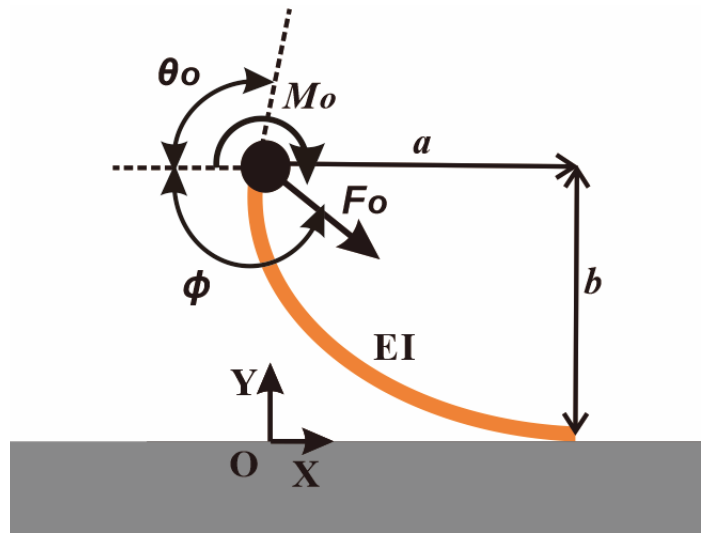

Figure 2. Diagram of large deflection cantilever's mechanical model.

Thus, we can model the deflection of the leg with an implicit method that can be incorporated into three large deflection equations, as shown below.

$$
\begin{gathered}
l_{2} \sqrt{F_{o} / 2 E I}=\frac{1}{2} \int_{0}^{\theta_{0}} \frac{d \theta}{\sqrt{\left[\cos \left(\theta_{0}+\Phi\right)-\cos (\theta+\Phi)\right]+M_{o}{ }^{2} / 2 E I F_{o}}} . \\
a=\frac{1}{2 \sqrt{F_{o} / 2 E I}} \int_{0}^{\theta_{0}} \frac{\cos (\theta) d \theta}{\sqrt{\left[\cos \left(\theta_{0}+\Phi\right)-\cos (\theta+\Phi)\right]+M_{o}{ }^{2} / 2 E I F_{o}}} . \\
b=\frac{1}{2 \sqrt{F_{o} / 2 E I}} \int_{0}^{\theta_{0}} \frac{\sin (\theta) d \theta}{\sqrt{\left[\cos \left(\theta_{0}+\Phi\right)-\cos (\theta+\Phi)\right]+M_{o}{ }^{2} / 2 E I F_{o}}} .
\end{gathered}
$$

where $a$ and $b$ denote the x-direction and y-direction displacements. $\theta_{0}$ denotes the angle of tangential vector at the end of the flipper leg. $F_{0}$, and $M_{0}$ denote the force and moment applied to the rotating tip, respectively. $\varphi$ is the direction of $F_{0} . l_{2}$ denotes the length of the bent compliant flipper leg. $E$ is Young's modulus of the leg and $I$ is the moment of inertia of the leg's cross section. Here we classified six independent variables into two groups, where the variables in the first group are $a, b$, and $\theta_{b}$, which stand for the posture and position of the end of the compliant leg; the variables in the second group include $F_{0}, M_{0}$ and $\varphi$, which stand for the load applied to the compliant leg. In these equations there are six independent variables, so more equations are needed as boundary conditions to obtain a solution, albeit the three equations could be solved with boundary conditions by numerical method because they are implicit.

\subsection{Model of a Leg Locomotion Platform}

As mentioned above, since the number of the independent variables is six, more equations are needed to obtain a solution for leg deformation. Here we considered the locomotion platform of a single compliant flipper leg to model the interactions between the flipper leg, the driving unit, and the ground. To obtain the boundary conditions during locomotion the motion of the compliant flipper leg was 
divided into three phases, as shown in Figure 3; the bending phase, the lifting phase, and the flying phase. In the bending phase the compliant leg begins to bend, and the driving unit is always in contact with the ground. In the lifting phase the driving unit begins to leave the ground while the surface beneath the leg is still in contact with the ground. In the flying phase the driving unit and the leg both leave the ground. This motion is similar but it is not strictly a free falling motion due to friction.

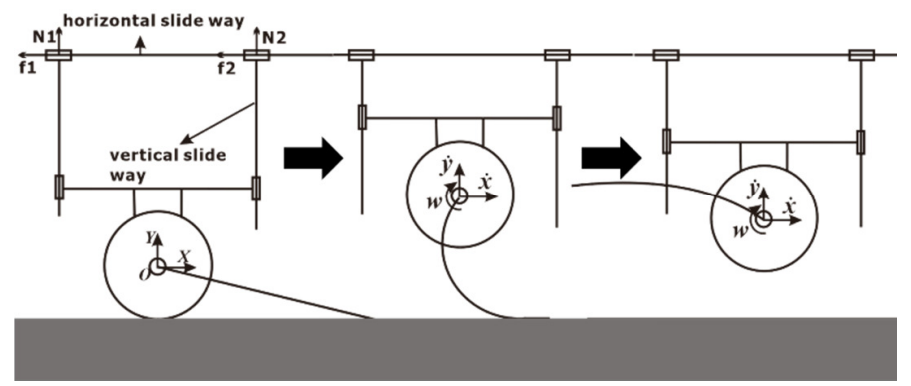

(a)

(b)

(c)

Figure 3. Three phases of the motion during one cycle of locomotion.

Fig. 4 is a mechanical diagram of the locomotion platform before the driving unit leaves the ground, that is, the bending phase. Here the driving unit remains stationary until $F_{y}$ is large enough to lift the driving unit up and enter the lifting phase.

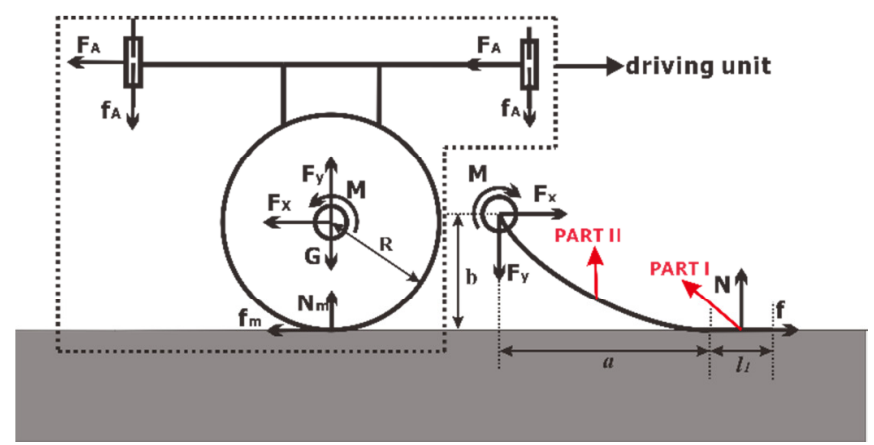

Figure 4. Diagram of the system before the driving unit leaves the ground (bending phase).

We considered that the leg bends slowly during the bending phase, so it can be regarded as a quasi-static process. In the figure, $F_{x}, F_{y}$, and $M$ are the x-axial load, the y-axial load, and the moment from motor applied to the upper end of the leg, respectively. $F_{A}$ and $f_{A}$ are the normal force and friction force between the sliders and vertical slide ways. The coefficient of friction of $F_{A}$ and $f_{A}$ is $\mu_{S}$, and $G$ stands for the gravity of the driving unit. Thus, the dynamical equations of the driving unit are as shown below:

$$
\begin{gathered}
F_{y}+N_{m}=G+2 f_{A} . \\
2 F_{A} h+f_{m} R=M . \\
f_{m}+2 F_{A}=F_{x} .
\end{gathered}
$$

The compliant flipper leg can be divided into two parts, Leg Part I and Leg Part II in Fig.4 so that three large deflection equations can be applied. Leg part I contacts the ground, while Leg part II denotes the transforming part of the leg. The friction force $f$ and normal force $N$ between the leg and the ground are almost equal to $F_{x}$ and $F_{y}$, and the moments are balance for leg part II are as listed below:

$$
N\left(a+0.5 l_{1}\right)+f b=M .
$$

where $l 1$ is the length of leg part I, and the coefficient of friction between the leg and the round is defined as $\mu_{d}$.

As analysed before, the driving unit leaves the ground when the y-axial component of $F_{0}$ is larger than the weight of the unit, and then the lifting phase begins. In this phase the dynamical model of the driving unit can be modelled using the following equations:

$$
\begin{gathered}
F_{x}-2 F_{A}=m \ddot{x} . \\
-2 f_{A}+F_{y}-m g=m \ddot{y} .
\end{gathered}
$$

where $m$ is the weight of the payload, $\ddot{x}$ and $\ddot{y}$ are the $\mathrm{x}$ axial and y axial accelerations, respectively. The bending of the leg is still regarded as a quasi-static process if the speed of rotation is low. The driving unit enters the flying phase when the length of leg part I becomes zero.

These equations are difficult to solve due to the integral and second derivative. To solve Equations 1-3 where the integral is involved, a searching method was used to look for solution within given errors. As for Equations 8-9 that contained a second derivative, we used an iterative algorithm for this calculation.

\subsection{Robot Locomotion Platform}

A platform for a robot with four legs has also been developed to explore the performance of the compliant flipper-leg as a propulsion unit for a complete robot. This platform has four yellow compliant legs made from polyurethane, as shown in Figure 5. The frame of this robot is $385 \times 620 \times 110 \mathrm{~mm}$, and it weighs $11.3 \mathrm{~kg}$. Yellow sponges are fixed to the bottom of the robot to absorb the shock when it collides with the floor to protect the mechanical structures, circuit boards, and motors. When the robot is stationary on the floor, Point $\mathrm{C}$ is $70 \mathrm{~mm}$ above the floor. Each leg is driven by a MAXON motor with an Elmo driving unit. The frame of the robot is assembled by 2020 aluminum profiles. The compliant leg is designed to be a simple rectangular block $315 \mathrm{~mm}$ long x $55 \mathrm{~mm}$ wide $\mathrm{x} 15 \mathrm{~mm}$ thick. 
The robot has a gait such that four legs are propelled simultaneously. When the robot is turned on, the legs bend the motor drives the robot up and then propels it forward. In the experiment, the forward speeds, fluctuations in height, and the cost of transport (COT) were recorded to evaluate how well the robot performed. COT is a non-dimensional value to evaluate the consumption of energy as the animals propel themselves along, or the robots are transported [28]. COT is also widely used to study the cost of robot's energy during locomotion [29],[30]. Here COT is defined as shown below:

$$
\operatorname{coT}=\frac{P_{i}}{W V}
$$

$P_{i}$ is the input power provided by the motor, $W$ is the gravity of the system, and $V$ is the average forward speed of the robot. COT is calculated for an entire stride, which means the legs experience motion in all three phases, in a period defined as $T$. Since the output power of the motors changes during locomotion, $P_{i}$ is defined as the mean output power of the motors that we record at a discrete time in a stride period $\mathrm{T}$ :

$$
P_{i}=\frac{\omega}{T} \sum_{T} M(t) \Delta t_{i}
$$

where $M(\mathrm{t})$ means the output torque of the motor at time $t$, and $\Delta t_{i}$ means the interval of time between two recorded torque values. By combining Equations 12 and 11, COT is finally formed as shown below:

$$
\mathrm{COT}=\frac{\omega \Sigma_{T} M(t) \Delta t_{i}}{W V T} .
$$

To calculate COT, we must obtain the robot's forward speed, and the motor's output torque and period of stride. We recorded the leg bending process and robot movement with a camera, so its forward speed and period time can be calculated. We obtained the motor's output torque by recording the current flowing through its coil, based on the relationship where the output torque is proportional to the current. Before commencing this experiment, a whiteboard with standardised grids, called a mask board, was place along a red line marked on the rubber floor. We then used a camera to photograph the mask board and then use it as background. We then remove the mask board and allowed the robot to walk along the red line. This red line is to ensure that the mask board and one side of robot's leg would be in the same vertical plane, and thus eliminate any error caused by the visual angle of the camera. The camera also recorded the movement of the robot as a video with 30 frames per second. After finishing the experiment, we extracted every frame from the video, and overlaid them with the background photo. By reading the position of the output axle of the motor marked as point $\mathrm{C}$ in Figure 5 (a) on the mask board, we obtained the trajectories and length of stride at while the motor was at different speeds of rotation. The period of stride $T$ is calculated from when the motor begins to rotate to when the robot falls down on the floor. Then its forward speed could be calculated using the length of stride and period of time. Every experiment was repeated three times under the same condition in order to obtain any errors.

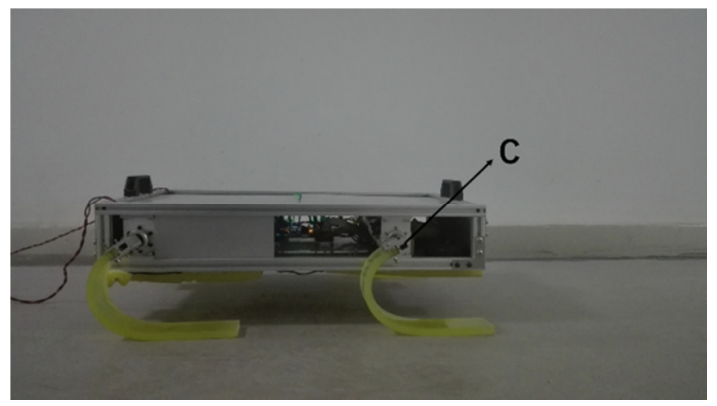

(a)

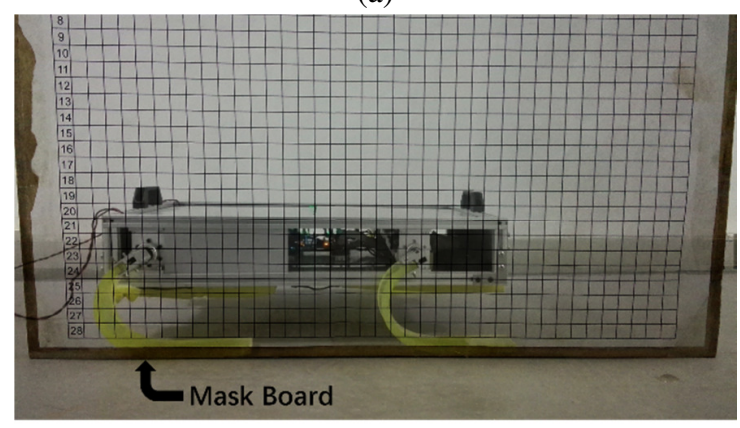

(b)

Figure 5. Four-leg-robot locomotion platform.

\section{Theoretical and Experimental Results}

With the platforms and models now developed, we can examine and explore the locomotion of the compliant flipper leg under various conditions.

\subsection{Results of Single-leg Platform}

Fig. 6 shows the trajectories of the compliant flipper leg at $0.5 \mathrm{rad} / \mathrm{s}$ speed of rotation in the simulations and experiments, respectively. The straight yellow bar represents the initial posture and position of the compliant flipper leg, and the black dots represent the centre of the motor's output axle. The trajectory can be divided into part I, part II, and part III. The result shows that the two trajectories coincided well for part I, and the simulated length of stride was almost the same as in the experiment. However, the trajectory from the simulation was higher than the experiment for part II, while Part III was close to falling freely. Both trajectories actually 
fell quickly, but in the simulation the driving unit fell faster than the other one. This inconsistency between the two trajectories from the simulation and experiment occurred because (1) Polyurethane is not an ideal linear material because its elastic modulus decreases with a large strain; (2) The inertial force of the driving unit was ignored because we assumed that the movement of the driving unit would be slow; (3) The coefficient between rubber material such as polyurethane and wooden ground may not be a constant value, an average measured value was used for the coefficient of friction. Despite the small difference in the trajectory between the simulation and the experiment, we concluded that the mechanical model can basically reflect the locomotion dynamics of the compliant flipper leg.

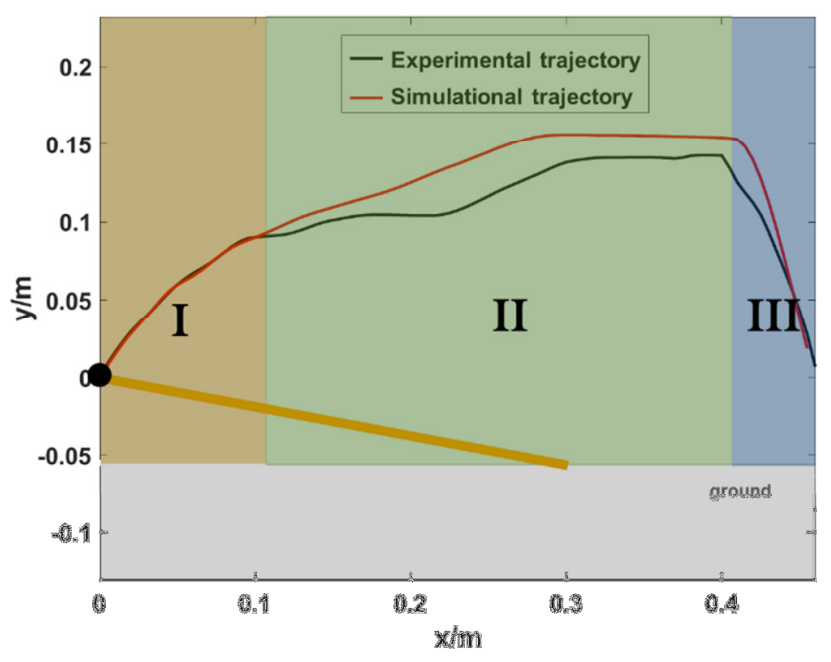

Figure 6. Trajectories of the compliant flipper leg.

Based on the mechanical analysis of a single-leg locomotion platform, the trajectories, forward speed $V_{\mathrm{x}}$ and fluctuating height $H$ of the driving unit were calculated with respect to five different rotation speeds $\omega$ of motor, as shown in Figure 7.

Figure 7 (a) shows the trajectories of the leg rotation axle during locomotion when the leg rotates at speeds of $0.5 \mathrm{rad} / \mathrm{s}$, $1 \mathrm{rad} / \mathrm{s}, 2 \mathrm{rad} / \mathrm{s}, 3 \mathrm{rad} / \mathrm{s}$, and $4 \mathrm{rad} / \mathrm{s}$, respectively. When the driving unit raised itself from the floor, the trajectories at lower rotating speeds of $0.5 \mathrm{rad} / \mathrm{s}, 1 \mathrm{rad} / \mathrm{s}$, and $2 \mathrm{rad} / \mathrm{s}$ were similar. The trajectories at $0.5 \mathrm{rad} / \mathrm{s}, 1 \mathrm{rad} / \mathrm{s}$ and $2 \mathrm{rad} / \mathrm{s}$ increased slowly until they were almost coincident at the first half part. These three trajectories also had a similar length of stride because the trajectory at $3 \mathrm{rad} / \mathrm{s}$ rises quickly and with a shorter length stride than those at lower rotating speeds. However, the trajectory at a rotating speed of $4 \mathrm{rad} / \mathrm{s}$ appears to be different because it rises very quickly and then drops down quickly; this was the shortest stride length in the trajectories at all five speeds. Figure 7 (b) and (c) presents the forward speed, the COT, and fluctuations in the height of the locomotion platform, respectively. Here, the forward speed $V_{\mathrm{x}}$ increased from $0.5 \mathrm{rad} / \mathrm{s}$ to $3 \mathrm{rad} / \mathrm{s}$ and then decreased at 4 $\mathrm{rad} / \mathrm{s}$. The fluctuations in height decreased at $0.5 \mathrm{rad} / \mathrm{s}$ to 3 $\mathrm{rad} / \mathrm{s}$ and then increased at $4 \mathrm{rad} / \mathrm{s}$. The highest forward speed and lowest fluctuations in height are both at $3 \mathrm{rad} / \mathrm{s}$. The dynamics of COT shows that it generally became larger at higher rotating speeds but experienced its lowest value at 2 $\mathrm{rad} / \mathrm{s}$. Moreover, COT changed slowly from $0.5 \mathrm{rad} / \mathrm{s}$ to 3 $\mathrm{rad} / \mathrm{s}$, and then went up quickly from $3 \mathrm{rad} / \mathrm{s}$ to $4 \mathrm{rad} / \mathrm{s}$. So, if the motor is allowed to rotate faster, a higher forward speed can be obtained without sacrificing energy efficiency, and if the motor rotates very quickly, forward speed decreases and the energy efficiency drops quickly.

It can be concluded from the above that the motions at 0.5 $\mathrm{rad} / \mathrm{s}, 1 \mathrm{rad} / \mathrm{s}, 2 \mathrm{rad} / \mathrm{s}$ and $3 \mathrm{rad} / \mathrm{s}$ were regular, but when $\omega$ was $4 \mathrm{rad} / \mathrm{s}$, the motion was out of the order. By observing the simulated animation, we found that the compliant leg slips badly on the ground, and it was this slippage that decreased the forward speed and COT; it can also shorten the length of stride that results in a lower forward speed.

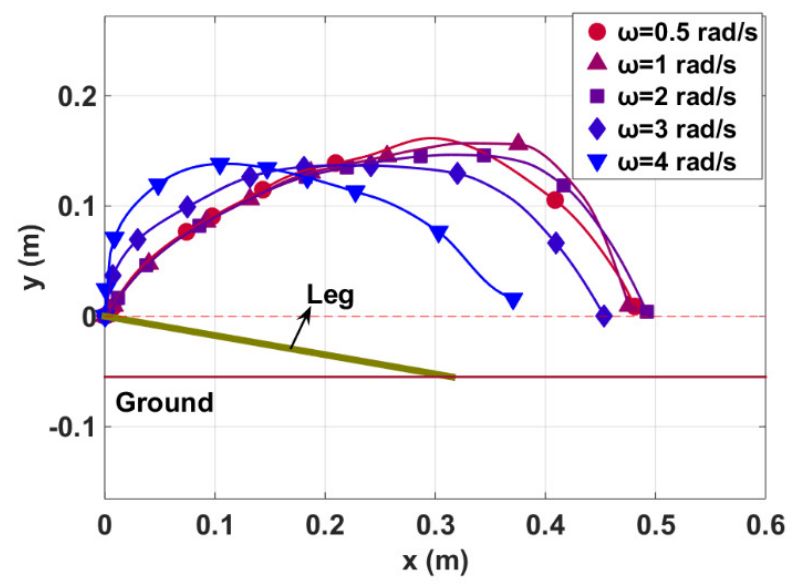

(a) 


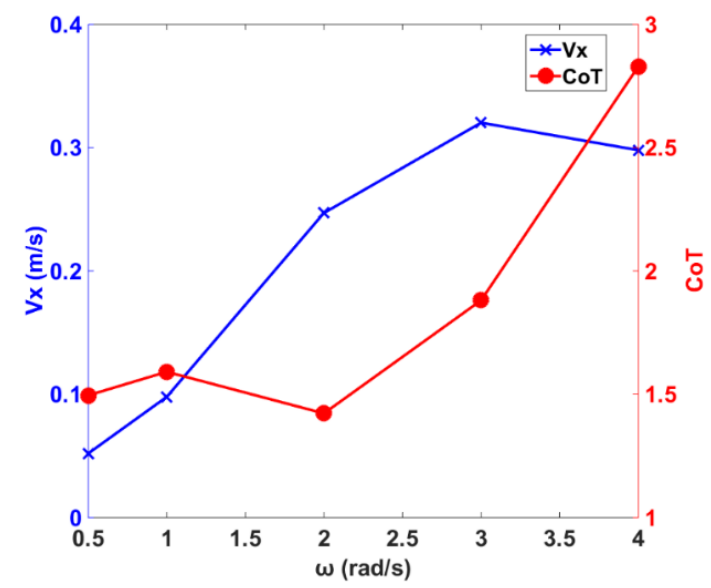

(b)

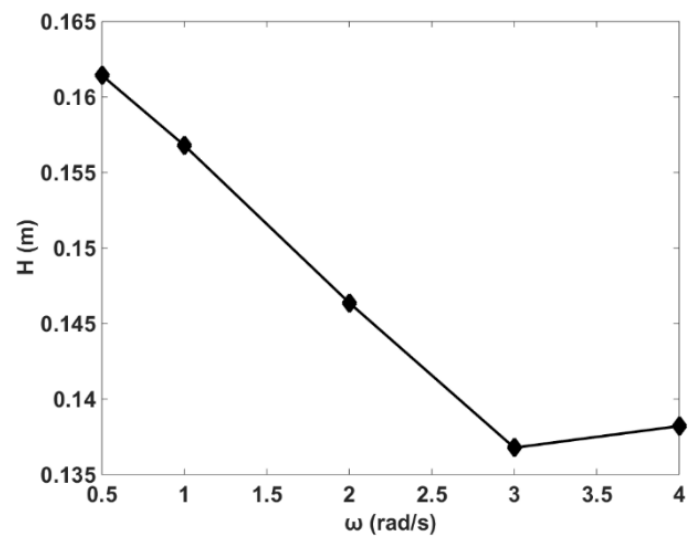

(c)

Figure 7. Simulation results of the single leg locomotion platform.

\subsection{Results of Four-leg-robot Platform}

The robot locomotion platform allows the locomotion of the compliant flipper leg to be examined experimentally. As mentioned before, we selected variable rotation speeds in the experiments. After considering the capability of the motors, a range of speed from $0.5 \mathrm{rad} / \mathrm{s}$ to $4 \mathrm{rad} / \mathrm{s}$ with just one stride was chosen for recording purposes. Their trajectories are shown in Figure 8 (a). All the trajectories are similar at 0.5 $\mathrm{rad} / \mathrm{s}, 1 \mathrm{rad} / \mathrm{s}, 2 \mathrm{rad} / \mathrm{s}$ and $3 \mathrm{rad} / \mathrm{s}$, but when the motor reached a rotating speed of $4 \mathrm{rad} / \mathrm{s}$, the trajectory is obviously higher than others when $\mathrm{x}$ is around $0.1 \mathrm{~m}$ and the length of stride becomes shorter. So at low speeds, the speed at which the motor rotates is not an important factor of trajectory because it only begins to differ at higher speeds. The video shows that at low speeds, the legs of the robot did not slip on the ground, but once the speed of rotation increased, so too did slippage.
In Figure 8 (c), fluctuations in the height of the robot increased slowly before reaching $3 \mathrm{rad} / \mathrm{s}$, but from $3 \mathrm{rad} / \mathrm{s}$ to $4 \mathrm{rad} / \mathrm{s}$, the height fluctuated faster due to slippage. Figure 8 (b) shows that the robot's forward speed varied with COT at five speeds ranging from $0.5 \mathrm{rad} / \mathrm{s}$ to $4 \mathrm{rad} / \mathrm{s}$. The chart representing forward speed shows that the forward speed $V_{\mathrm{x}}$ is strictly linear, and thus the forward speed of robots propelled by compliant legs on terrain is approximately proportional to the rotation speed of the motor. The proportional factor was $75.6 \mathrm{~mm} /(\mathrm{rad} / \mathrm{s})$, so the maximum forward speed can reach $0.3 \mathrm{~m} / \mathrm{s}$ at $4 \mathrm{rad} / \mathrm{s}$, which is 0.48 body lengths per second. Another chart in Figure 8 (b) shows the cost of transport where As COT increased with almost all rotating speeds the compliant leg experienced a subsequent decrease in its power efficiency. As with the fluctuations in height, COT rose faster from $3 \mathrm{rad} / \mathrm{s}$ to $4 \mathrm{rad} / \mathrm{s}$ because more energy dissipated in dynamical friction between the leg and the ground. Though a higher rotation speed leads to a higher forward speed, slippage occurs at this situation, which leads to a sharp decline of power efficiency

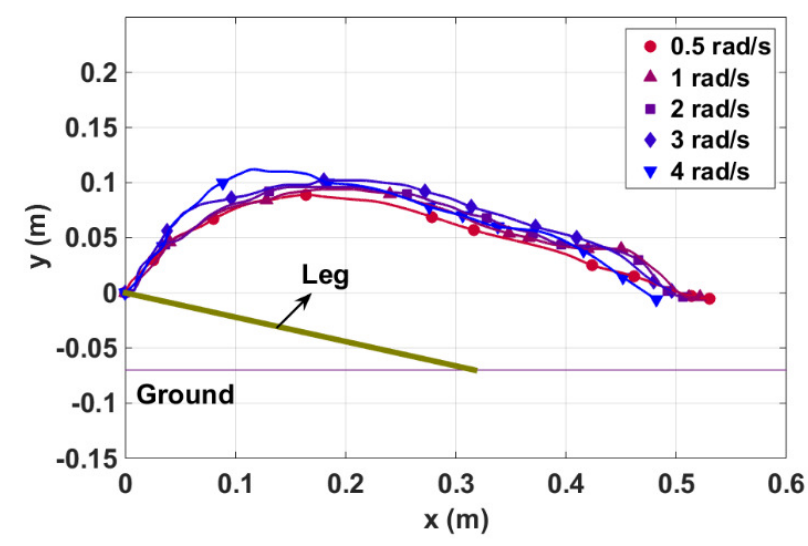

(a)

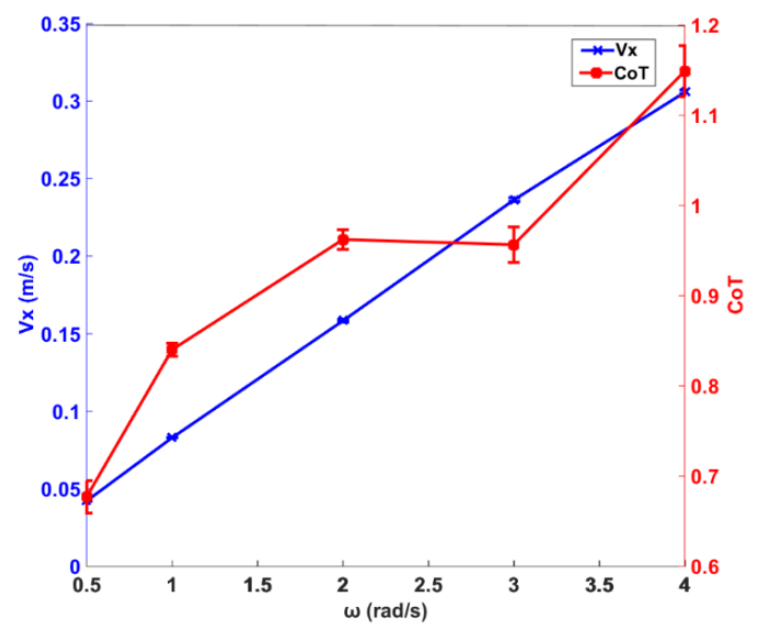

(b) 


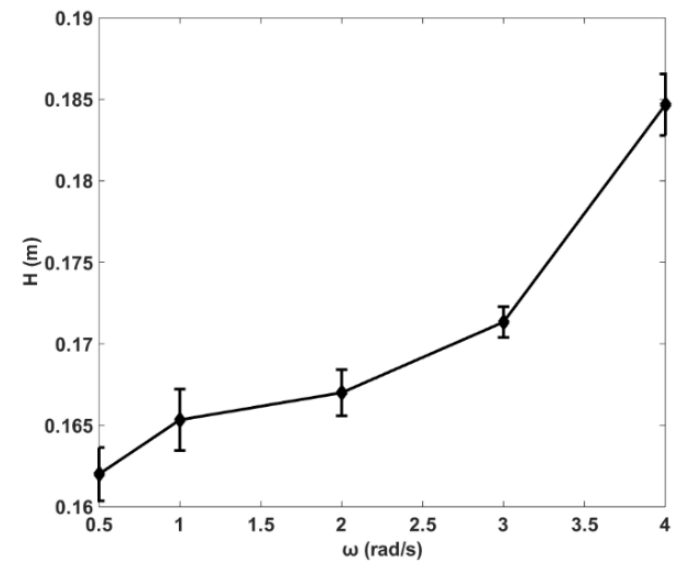

(c)

Figure 8. Experimental result of robot locomotion platform.

\subsection{Special Design of Compliant Flipper Legs}

Now that the mechanical model has been developed, the design of the complaint flipper legs can be examined in order to obtain a high locomotion performance. By observing animals with long compliant legs we find that almost all legs have a slope from the root to the end tip, and the tip of the root is thicker than the tip at the end, just like leg I in Figure 9 (a). In order to validate the advantages of a flipper leg with such a shape, we constructed two different legs; one leg has the same thickness along its length, while the other was thinner at the root tip and thicker at the end tip. These two legs are called leg II and leg III, respectively, as shown in Figure 9(a). Thus, we now have three leg shapes with different slopes, so the locomotion on terrain can now be compared.

We calculated the trajectories $V_{\mathrm{x}}$, and COT based on a single leg platform with three different legs, and presented the results in Figure 9(a) and (b). The figure shows that leg I has the highest forward speed and the lowest COT, so a leg shaped like Leg I was better than the other two. That leg I had the best performance coincides with the cases in nature, where for instance, fish are stiffer in their anterior region than at their posterior region, so leg 1 was better able to reproduce the kinematics of fish swimming freely [31]. The trajectory of Leg III is lowest and has an obvious valley that was probably caused by energy accumulation and releasing as the compliant leg was bending. Since leg III has a thinner root tip and a strong end tip, when the leg begins to bend, more energy is stored in the root which causes more the root to bend more, and that results in a lower trajectory. The stored energy is released when it enters the flying phase because the end tip is strong enough, and it has bent less and thus can generate more propulsive force, which let the driving unit go up obviously and then a valley appears.

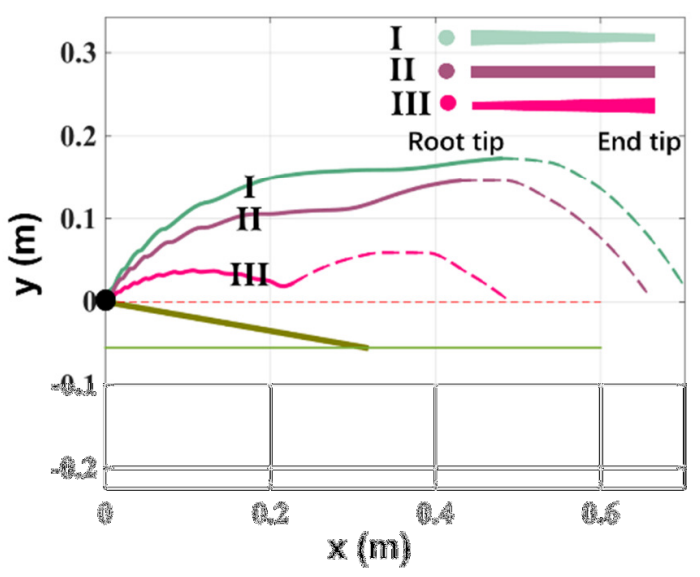

(a)

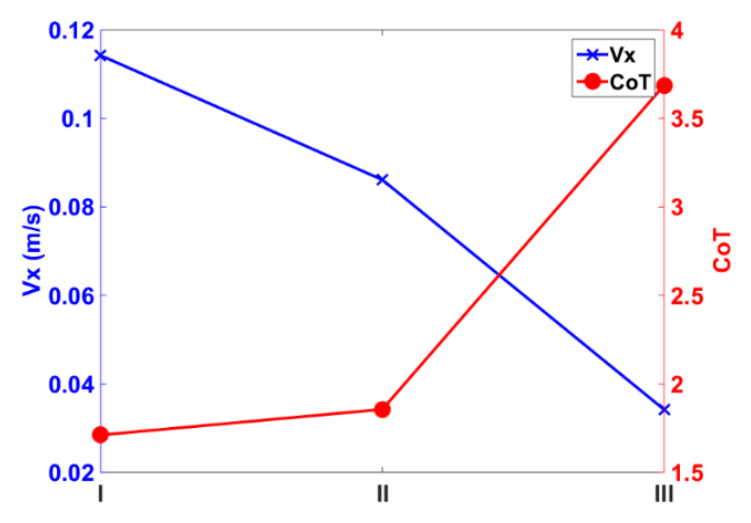

(b)

Figure 9. Special design of the complaint flipper legs.

\subsection{Discussion}

In the above section, we presented the results from simulations and experiments for a single-leg platform and experiments for four-leg-robot platform, including their trajectories, forward speed, fluctuations in height, and COT versus various output rotation speed of the motor unit.

It is necessary to point out why two different platforms were used to explore the performance of the compliant legs, and compare the results of two different platforms. The description of the single-leg platform in Section 2.1 indicates that the horizontal load, including the vertical slide ways and some other parts, weight almost $12.36 \mathrm{~kg}$, which is much larger than the $2 \mathrm{~kg}$ vertical load. There was no situation where 
a compliant leg was used in a robot, even though a a multiple-leg platform was necessary. However, a four-leg-robot platform is difficult to model because the two front legs have a different mechanical motion to the two back legs. We therefore used a single-leg platform to simulate a compliant flipper leg, and a four-leg-robot platform to examine how this kind of flipper leg would perform.

Although these two platforms were built for different purposes, there are still some interesting results from a comparison of locomotion. When the motion of the driving unit in the single-leg platform finishes its lifting phase and enters the flying phase, the shift of motion is more obvious than in four-leg-robot platform because the weight of vertical slide ways in the single-leg platform is enough to generate considerable inertia which causes the driving unit to quickly decelerate, and the leg slip on the ground easily. For that reason, the driving unit's length of stride decreases at a rotation speed of $4 \mathrm{rad} / \mathrm{s}$ compared to slower speeds. Alternatively, forward speed increases when speed of rotation of the robot's locomotion platform increased from $0.5 \mathrm{rad} / \mathrm{s}$ to $4 \mathrm{rad} / \mathrm{s}$.

\section{Conclusions}

The purpose of this paper is to present a theoretical and experimental analysis of a compliant straight flipper-leg during terrestrial locomotion. Through the results from a single-leg and a four-leg-robot locomotion platform, we found that the best forward speed is linked to a certain rotation speed of the motor; if the motor rotates too fast, slippage between the leg and the ground occurs. To analyse propulsive efficiency, we calculated COT and found that a lower speed of rotation is better because once the legs rub on the floor, COT increases quickly. The locomotion performance of a four-leg robot reveals that these compliant legs can be applied to robots. Moreover, we compared three kinds of legs with different slopes and found that Leg I performed best at forward speed and propulsiveefficiency. Leg I has a slope where its thickness decreases from the root tip to the end tip, which is similar to animal flippers in nature. Future work will focus on a theoretical and experimental exploration of compliant flipper-legs walking on other terrain such as soil and sand.

\section{Reference}

[1] Boxerbaum, A. S., Werk, P., Quinn, R. D., \& Vaidyanathan, R. (2005, July). Design of an autonomous amphibious robot for surf zone operation: part I mechanical design for multi-mode mobility. In Advanced Intelligent Mechatronics. Proceedings, 2005 IEEE/ASME International Conference on (pp. 1459-1464). IEEE.

[2] Ijspeert, A. J., \& Cabelguen, J. M. (2014). Control of Aquatic and Terrestrial Gaits in Salamander. In Encyclopedia of Computational Neuroscience (pp. 1-9). Springer New York.

[3] Ijspeert, A. J., Crespi, A., Ryczko, D., \& Cabelguen, J. M. (2007). From swimming to walking with a salamander robot driven by a spinal cord model. science,315(5817), 1416-1420.

[4] Hirose, S. Biologically Inspired Robots: Snake-Like Locomotors and Manipulators, Oxford University Press, Oxford (1993).

[5] Low, K. H., Zhou, C., Ong, T. W., \& Yu, J. (2007, December). Modular design and initial gait study of an amphibian robotic turtle. In Robotics and Biomimetics, 2007. ROBIO 2007. IEEE International Conference on (pp. 535-540). IEEE.

[6] Liang, X., Xu, M., Xu, L., Liu, P., Ren, X., Kong, Z., ... \& Zhang, S. (2012, October). The amphihex: A novel amphibious robot with transformable leg-flipper composite propulsion mechanism. In Intelligent Robots and Systems (IROS), 2012 IEEE/RSJ International Conference on (pp. 3667-3672). IEEE.

[7] Zhang, S., Liang, X., Xu, L., \& Xu, M. (2013). Initial development of a novel amphibious robot with transformable fin-leg composite propulsion mechanisms. Journal of Bionic Engineering, 10(4), 434-445.

[8] Zhang, S., Zhou, Y., Xu, M., Liang, X., Liu, J., \& Yang, J. AmphiHex-I: Locomotory Performance in Amphibious Environments with Specially Designed Transformable Flipper-legs.

[9] Calisti, M., Corucci, F., Arienti, A., \& Laschi, C. (2015). Dynamics of underwater legged locomotion: modeling and experiments on an octopus-inspired robot. Bioinspiration \& biomimetics, 10(4), 046012.

[10] Çulha, U., \& Iida, F. (2016). Enhancement of finger motion range with compliant anthropomorphic joint design. Bioinspiration \& biomimetics, 11(2), 026001 .

[11] Umedachi, T., Vikas, V., \& Trimmer, B. A. (2016). Softworms: the design and control of non-pneumatic, 3D-printed, deformable robots. Bioinspiration \& Biomimetics, 11(2), 025001.

[12] Wang, X., Chen, Z., Fang, T., Xu, M., \& Zhang, S. (2016). Experimental Study on The Compliant 
Straight Leg for Mobile Robots. In ASSISTIVE ROBOTICS: Proceedings of the 18th International Conference on CLAWAR 2015 (pp. 494-501).

[13] El Daou, H., Salumäe, T., Chambers, L. D., Megill, W. M., \& Kruusmaa, M. (2014). Modelling of a biologically inspired robotic fish driven by compliant parts. Bioinspiration \& biomimetics, 9(1), 016010.

[14] Park, Y. J., Huh, T. M., Park, D., \& Cho, K. J. (2014). Design of a variable-stiffness flapping mechanism for maximizing the thrust of a bio-inspired underwater robot. Bioinspiration \& biomimetics, 9(3), 036002.

[15] Kancharala, A. K., \& Philen, M. K. (2014). Study of compliant fin and compliant joint stiffness on propulsive performance: theory and experiments. Bioinspiration \& biomimetics, 9(3), 036011.

[16] Kim, H. J., Song, S. H., \& Ahn, S. H. (2013). A turtle-like swimming robot using a smart soft composite (SSC) structure. Smart Materials and Structures, 22(1), 014007.

[17] Otake, M., Kagami, Y., Inaba, M., \& Inoue, H. (2002). Motion design of a starfish-shaped gel robot made of electro-active polymer gel. Robotics and Autonomous Systems, 40(2), 185-191.

[18] M. H. Raibert, Legged Robots That Balance. Cambridge, MA: MIT Press, 1986.

[19] Ahmadi, M., \& Buehler, M. (1997). Stable control of a simulated one-legged running robot with hip and leg compliance. Robotics and Automation, IEEE Transactions on, 13(1), 96-104.

[20] Howell, Larry L. Compliant mechanisms. John Wiley \& Sons, New York, 2001.

[21] Howell, L. L., \& Midha, A. (1995). Parametric deflection approximations for end-loaded, large-deflection beams in compliant mechanisms. Journal of Mechanical Design, 117(1), 156-165.

[22] Park, H. S., \& Sitti, M. (2009, October). Compliant footpad design analysis for a bio-inspired quadruped amphibious robot. In Intelligent Robots and Systems, 2009. IROS 2009. IEEE/RSJ International Conference on (pp. 645-651). IEEE.

[23] Hai-Jun Su, (2009, May), A Pseudo-Rigid-Body 3R Model for Determining Large Deflection of Cantilever Beams Subject to Tip Loads, Journal of Mechanisms and Robotics, 1(2), 1-9

[24] Huang, K. J., Huang, C. K., \& Lin, P. C. (2014). A simple running model with rolling contact and its role as a template for dynamic locomotion on a hexapod robot. Bioinspiration \& biomimetics, 9(4), 046004.

[25] Galloway, K. C., Clark, J. E., \& Koditschek, D. E. (2013). Variable stiffness legs for robust, efficient, and stable dynamic running. Journal of Mechanisms and Robotics, 5(1), 011009.

[26] Xia, K. (2011). Finite element modeling of tire/terrain interaction: Application to predicting soil compaction and tire mobility. Journal of Terramechanics, 48(2), 113-123.

[27] Ozaki, S., \& Kondo, W. (2016). Finite element analysis of tire traveling performance using anisotropic frictional interaction model. Journal of Terramechanics, 64, 1-9.

[28] Tucker, V. A. (1975). The energetic cost of moving about: walking and running are extremely inefficient forms of locomotion. Much greater efficiency is achieved by birds, fish-and bicyclists. American Scientist, 413-419.

[29] Cao, Q., \& Poulakakis, I. (2015). On the energetics of quadrupedal running: predicting the metabolic cost of transport via a compliant-torso model. Bioinspiration \& biomimetics, 10(5), 056008.

[30] Seok, S., Wang, A., Chuah, M. Y., Otten, D., Lang, J., \& Kim, S. (2013, May). Design principles for highly efficient quadrupeds and implementation on the MIT Cheetah robot. In Robotics and Automation (ICRA), 2013 IEEE International Conference on (pp. 3307-3312). IEEE.

[31] Kelsey N Lucas, Patrick J M Thornycroft, Brad J Gemmell, Sean P Colin, John H Costello and George V Lauder. Effects of non-uniform stiffness on the swimming performance of a passively-flexing, fish-like foil model. 2015 $\begin{array}{llll}\text { Bioinspir. } & \text { Biomim. } & 10 & 056019\end{array}$ doi:10.1088/1748-3190/10/5/05601 